\title{
ON THE PHASE PORTRAIT OF THE FAST FILTERING ALGORITHMS*
}

\author{
YISHAO $\mathrm{ZHOU}^{1}$
}

\begin{abstract}
Fast filtering algorithms arising from linear filtering and estimation are nonlinear dynamical systems whose initial values are the statistics of the observation process. In this paper, we give a fairly complete description of the phase portrait for such nonlinear dynamical systems, as well as a special type of naturally related matrix Riccati equation.

Résumé. Les algorithmes rapides de filtrage provenant du filtrage linéaire sont des systèmes dynamiques non linéaires dont les valeurs initiales sont les statistiques du processus d'observation. Dans ce papier, on donne une description assez complète du portrait de phase de ces systèmes dynamiques nonlinéaires, ainsi que d'une équation particulière de Riccati matricielle associée.
\end{abstract}

AMS Subject Classification. 93E11, 93B27, 58F40.

Received March 18, 1999. Revised October 7, 1999.

\section{INTRODUCTION}

The main purpose of this paper is to determine the complete phase portrait of the "fast filtering algorithm" used in linear filtering and estimation, which incorporates the statistics of the observation process as initial conditions for a dynamical system.

It was shown by Byrnes et al. [5] that, for the first-order filtering problems, solutions of the fast filtering algorithm, for nonclassical initial conditions, can

(i) evolve in unbounded, complicated excursions, or

(ii) exhibit periodic behavior of every period, or

(iii) converge to a classical limit,

and a complete phase-portrait was given. In Byrnes et al. [7] this result was generalized to systems of arbitrarily order essentially only for dynamical behavior of type (iii). There, for a scalar observation process, necessary and sufficient conditions for the Kalman filter to converge are derived using methods from stochastic systems and from nonlinear dynamics - especially the use of stable, unstable and center manifolds.

The major motivation for understanding the dynamics of this type of fast filtering algorithm, in addition to its own interests in nature of mathematics, lies on a series of recent work on rational covariance extension problem by Byrnes, Lindquist et al. (e.g. $[3,4,9,10])$. To the author's best knowledge these works deal with the strictly positive real data. That is, they solved the rational covariance extension problem in terms of rational functions

Keywords and phrases: Fast filtering algorithms, Riccati equations, Kalman filtering, nonlinear dynamical systems.

* This work was supported in part by Swedish Natural Science Research Council, NFR.

${ }^{1}$ Department of Mathematics, Stockholm University, 10691 Stockholm, Sweden; e-mail: yishao@matematik.su.se

(c) EDP Sciences, SMAI 1999 
which interpolate the data which have all poles inside the unit circle, and which have a strictly positive real-part on the unit circle. In certain applications of signal processing, it is desirable to allow zeros of the shaping filter which are either very close to, or lie on, the unit circle. Since one of the main results begins with the observation that filtering and interpolation define two "dual" or "complementary" decompositions of $\mathcal{P}_{n}$, the space of strictly positive real rational functions of degree at most $n$. Roughly speaking, the fast filtering algorithm defined on this space partition $\mathcal{P}_{n}$ into leaves of a foliation where the leaves consist of the stable manifolds of the filtering algorithms. On the other hand, each choice of "window", consisting of the first $n$ correlation coefficients or equivalently Schur parameters, also defines a leaf of a second foliation of $\mathcal{P}_{n}$. As we shall see later the filtering algorithm is not structurally stable when there is a lack of strictly positive realness, we believe that a complete phase portrait of this nonlinear dynamical system will give more insight for problems unsolved in the work on rational covariance extension problem.

In this paper, we attempt to describe, for $n$-th order filtering problems phase portraits of the fast filtering algorithm, as well as a special type of matrix Riccati equation. The analysis is akin to that used in describing the phase portrait of Riccati differential equation, see Shayman [23]. However, the results are quite different. The key issue here is to analyze the fast filtering algorithm, or Riccati equation when the relevant positivity conditions (in the context of spectral factorization) are not necessarily satisfied. In the language of Riccati equation theory, this is equivalent to say that the Hamiltonian matrix has eigenvalues on the imaginary axis. Another difference is that the orbit generated by the nonlinear system is in general not almost periodic. This is in sharp contrast to that of Riccati differential equation.

Theorem 7.1 in [7] shows that the abovementioned pseudo-polynomial being sign definite on the unit circle guarantees the convergence of the fast filtering algorithm. This paper is devoted to the situation when the pseudo-polynomial is sign indefinite, which, as we shall see, leads to discussion of dynamical behavior of type (ii).

The paper is organized as follows. In Section 2, we set notations and recall preliminary results needed throughout the paper. Then, we discuss heuristically on what will happen if certain positivity conditions are not satisfied in Section 3. The aim of this discussion is to giving a rough picture of the phase portrait of the fast filtering algorithm. Thereafter in Section 4, we determine the phase portrait of the fast filtering algorithm in a "generic" case. The proofs of statements in this section are given in Section 5. The purpose of Section 6 is to show that the phase portrait of the filtering algorithm can be described in a combinatorial way in terms of the rational dependence of the arguments for the zeros of the the pseudo-polynomial. The analysis is number theoretic. Since the filtering algorithm is an alternative method to compute Kalman gain, which can also be calculated through matrix Riccati equation, we are able to determine phase portraits in Section 7 for the Riccati equation corresponding to the filtering problem. The representative phase portraits are illustrated in the paper. We conclude the paper in Section 8 with a series of remarks and comments on our results, as well as weakness of our analysis.

\section{Preliminaries}

In this section, we shall fix some notations and recall some preliminary results needed in the sequel. Let $\left\{y_{0}, y_{1}, y_{2}, \ldots\right\}$ be a scalar stationary stochastic process that is the output of a linear, finite-dimensional stochastic system driven by white noise, i.e.

$$
\left\{\begin{aligned}
x_{t+1} & =F x_{t}+v_{t}, \\
y_{t} & =h^{\prime} x_{t}+w_{t} .
\end{aligned}\right.
$$

It is well-known that the minimum variance estimate $\hat{x}_{t}$ of the current state $x_{t}$ of the system is generated by the Kalman filter

$$
\hat{x}_{t+1}=F \hat{x}_{t}+k_{t}\left(y_{t}-h^{\prime} \hat{x}_{t}\right) ; \quad \hat{x}_{0}=0
$$


and the gain

$$
k_{t}=\left(1-h^{\prime} P_{t} h\right)^{-1}\left(g-F P_{t} h\right)
$$

is determined by solving the Riccati equation

$$
P_{t+1}=F P_{t} F^{\prime}+\left(g-F P_{t} h\right)\left(1-h^{\prime} P_{t} h\right)^{-1}\left(g-F P_{t} h\right)^{\prime}
$$

with $P_{0}=0$, where $g:=\mathrm{E}\left\{x_{t+1} y_{t}\right\}$. Let $v(z)$ be a proper rational function of degree $n$ with a minimal realization

$$
v(z)=\frac{1}{2}+h^{\prime}(z I-F)^{-1} g .
$$

Denote $v(z):=b(z) / a(z)$. If $v(z)$ is positive real ${ }^{1}$, then $P_{t}$ tends to the stable equilibrium of (2.4) monotonically [11]. Without loss of generality, we take $\left(h^{\prime}, F\right)$ in the observer canonical form. In [7], it was shown that $k_{t}$ can be computed by a reformulation of the fast filtering algorithm (see more detail in Lindquist $[18,19]$ ), i.e.

$$
k_{t}=\frac{1}{2}[b(t)-a(t)]-a(0)
$$

where $a(0)=a$ is the vector from the matrix $F$, which can be taken as the companion matrix $F=J-a h^{\prime}$ with $J$ being a shift matrix, and $a(t)$ and $b(t)$ can be computed by

$$
\begin{aligned}
& a(t)=\varphi_{n}(\gamma(t))+\Phi_{n}(\gamma(t)) \alpha(t), \\
& b(t)=\psi_{n}(\gamma(t))+\Psi_{n}(\gamma(t)) \alpha(t),
\end{aligned}
$$

and

$$
\begin{aligned}
\alpha(t+1) & =A(\gamma(t)) \alpha(t), & & \alpha(0)=\alpha \\
\gamma(t+1) & =G(\alpha(t+1)) \gamma(t), & & \gamma(0)=\gamma
\end{aligned}
$$

initiated at $(\alpha, \gamma)$ evolves on an invariant manifold, $X_{D}$, the level sets of the function $h_{i}: \mathbb{R}^{2 n} \rightarrow \mathbb{R}^{n}$ to be made clear in a moment, and

$$
\begin{aligned}
\Phi_{n+1} & =\left[\begin{array}{ccccc}
1 & & & & \\
\varphi_{n 1} & 1 & & & \\
\varphi_{n 2} & \varphi_{n-1,2} & 1 & & \\
\vdots & \vdots & \vdots & \ddots & \\
\varphi_{n n} & \varphi_{n-1, n-1} & \varphi_{n-2, n-2} & \cdots & 1
\end{array}\right] \\
\Psi_{n+1} & =\left[\begin{array}{ccccc}
1 & & & & \\
\psi_{n 1} & 1 & & & \\
\psi_{n 2} & \psi_{n-1,2} & 1 & & \\
\vdots & \vdots & \vdots & \ddots & \\
\psi_{n n} & \psi_{n-1, n-1} & \psi_{n-2, n-2} & \cdots & 1
\end{array}\right]
\end{aligned}
$$

\footnotetext{
${ }^{1} \mathrm{~A}$ function $v(z)$ is positive real if it is analytic for $|z| \geq 1$ and satisfies $v(z)+v(1 / z) \geq 0$ at each point of the unit circle where $v$ has no pole.
} 
The matrices $A$ and $G$ are defined by

$$
\begin{aligned}
& A(\gamma)=\left[\begin{array}{cccc}
\frac{1}{1-\gamma_{n-1}^{2}} & \frac{\gamma_{n-1} \gamma_{n-2}}{\left(1-\gamma_{n-1}^{2}\right)\left(1-\gamma_{n-2}^{2}\right)} & \cdots & \frac{\gamma_{n-1} \gamma_{0}}{\left(1-\gamma_{n-1}^{2}\right) \cdots\left(1-\gamma_{0}^{2}\right)} \\
0 & \frac{1}{1-\gamma_{n-2}^{2}} & \cdots & \frac{\gamma_{n-2} \gamma_{0}}{\left(1-\gamma_{n-2}^{2}\right) \cdots\left(1-\gamma_{0}^{2}\right)} \\
\vdots & \vdots & \ddots & \vdots \\
0 & 0 & \cdots & \frac{1}{1-\gamma_{0}^{2}}
\end{array}\right] \text {, } \\
& G(\alpha)=\left[\begin{array}{ccccc}
0 & 1 & 0 & \cdots & 0 \\
0 & 0 & 1 & \cdots & 0 \\
\vdots & \vdots & \vdots & \ddots & \vdots \\
0 & 0 & 0 & \cdots & 1 \\
-\alpha_{n} & -\alpha_{n-1} & -\alpha_{n-2} & \cdots & -\alpha_{1}
\end{array}\right] \text {. }
\end{aligned}
$$

The issue addressed in this paper is complete phase portraits of the nonlinear equation (2.7), as well as phase portraits of the related Riccati equation defined by $(2.4)$,

Now let $D(z, 1 / z):=\frac{1}{2}[a(z) b(1 / z)+a(1 / z) b(z)]$. The symmetric pseudo-polynomial $D$ is determined by the initial condition $(\alpha, \gamma)$ in a manner described by the following lemma.

Lemma 2.1. [7] Let $D\left(z, z^{-1}\right)$ be the pseudo-polynomial corresponding to the initial condition $(\alpha, \gamma)$. Then

$$
d_{0}=\alpha_{n}^{2}+r_{1} \alpha_{n-1}^{2}+\cdots+r_{n}
$$

where $r_{1}, r_{2}, \ldots, r_{n}$ are defined by $r_{t+1}=\left(1-\gamma_{t}^{2}\right) r_{t} ; r_{0}=1$, and $d_{i}:=d_{i}^{(n)}(\alpha, \gamma)$ for $i=1,2, \ldots, n$, where $d_{i}^{(n)}$ is determined recursively by

$$
\begin{aligned}
& d_{1}^{(1)}\left(\alpha_{1}, \gamma_{0}\right)=\alpha_{1} ; \\
& d_{i}^{(k)}\left(\alpha_{1}, \ldots, \alpha_{k}, \gamma_{0}, \ldots, \gamma_{k-1}\right)=\left(1-\gamma_{k-i-1}^{2}\right) d_{i}^{(k-1)}\left(\alpha_{1}, \ldots, \alpha_{k-1}, \gamma_{1}, \ldots, \gamma_{k-1}\right) \\
& \quad+\alpha_{k} \sum_{j=1}^{k} \alpha_{k-j} \pi_{j, j-i}, \quad \text { for } i=1,2, \ldots, k-1 ; \\
& d_{k}^{(k)}\left(\alpha_{1}, \ldots, \alpha_{k}, \gamma_{0}, \ldots, \gamma_{k-1}\right)=\alpha_{k} ;
\end{aligned}
$$

where $\left\{\pi_{j l}\right\}$ are the coefficients of the polynomials

$$
\pi_{j}(z)=z^{j}+\pi_{j 1} z^{j-1}+\cdots+\pi_{j j}
$$

generated by the polynomial recursion

$$
\left\{\begin{array}{l}
\pi_{t+1}(z)=(1+z) \pi_{t}(z)+\left(\gamma_{t} \gamma_{t-1}-1\right) z \pi_{t-1}(z) \\
\pi_{0}=1, \quad \pi_{1}(z)=z
\end{array}\right.
$$

and $\pi_{j i}=0$ for $i>j$. Moreover, if $\gamma_{k}^{2} \neq 1$ for $k=0,1, \ldots, n-1$, then at least one of the coefficients $d_{0}, d_{1}, \ldots, d_{n}$ of the pseudo-polynomial $D\left(z, z^{-1}\right)$ is nonzero.

It was also shown in [7] that

$$
r_{t} d_{i}(\alpha(t), \gamma(t))=d_{i}(\alpha(0), \gamma(0)) \quad i=0,1,2, \ldots, n
$$


for all $t \in \mathbb{Z}$ along the trajectory of the dynamical system (2.7). Hence the $n+1$ functions $d_{i}(\alpha, \gamma)$, $i=0,1, \ldots, n-1$ defined in Lemma 2.1, are invariant under the evolution of (2.7) up to a (common) scaling factor; i.e. these $(n+1)$ functions are projectively invariant. We can obtain $n$ invariant quantities, either by viewing the pseudo-polynomial, in terms of homogeneous coordinates, as a point in $\mathbb{P}^{n}$ (see [22]), or equivalently by dividing each of the $(n+1)$ equations in $(2.12)$ by any one of the $(n+1)$ functions which is nonzero (by Lem. 2.1, there is always one), obtaining rational functions having values independent of $r_{t}$ and hence depending only on $(\alpha, \gamma)$. That is, we can view the pseudo-polynomial $D$ either as determining $(n+1)$ projectively invariant functions $T_{1}, \ldots T_{n+1}$ or as determining a map $\bar{T}$ to $\mathbb{P}^{n}$ :

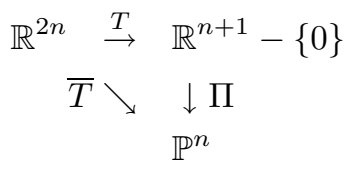

where $T=\left(T_{1}, \ldots, T_{n+1}\right)$ and $\bar{T}=\Pi \circ T$ where

$$
\Pi\left(x_{1}, \ldots, x_{n+1}\right)=\left[x_{1}, \ldots, x_{n+1}\right] .
$$

Indeed, this analytic set is a smooth $n$-manifold at a point $(\alpha, \gamma)$ provided $\left.\operatorname{Jac} T\right|_{(\alpha, \gamma)}$ has an $n$-dimensional kernel.

Finally, we review how the Riccati equation (2.4) is extended to the Lagrange-Grassmann manifold, denoted by $\mathcal{L}(n)$. Let $J$ denote the $2 n \times 2 n$ matrix $\left[\begin{array}{cc}0 & I \\ -I & 0\end{array}\right]$, and $\omega$ is a nondegenerate bilinear skew symmetric form on $\mathbb{R}^{2 n \times 2 n}$, defined by $\omega(x, y)=x^{\prime} J y$. Then, $\mathcal{L}(n)$ is defined to be a subset $\left\{V \in G^{n}\left(\mathbb{R}^{2 n}\right): J(V)=V^{\perp}\right\}$. We assume that the standard inner product is assigned to $\mathbb{R}^{2 n}$ (of any dimension) is Lagrangian if and only if $J(V) \perp V$. Let $\operatorname{Sp}(n, \mathbb{R})$ denote the symplectic group, that is, $\operatorname{Sp}(n, \mathbb{R})=\left\{P \in \operatorname{Gl}(2 n, \mathbb{R}): P^{\prime} J P=J\right\}$. The Lie algebra associated with $\operatorname{Sp}(n, \mathbb{R})$ can be shown to be $\operatorname{sp}(n, \mathbb{R})=\left\{H \in \mathbb{R}^{2 n \times 2 n}: J H+H J^{\prime}=0\right\}$. A symplectic basis for $\mathbb{R}^{2 n}$ is a basis $v_{1}, v_{2}, \ldots, v_{2 n}$ such that $\omega\left(v_{i}, v_{j}\right)=J_{i j}$, where $J=\left(J_{i j}\right)$. A symplectic space always admits a symplectic basis. If $V \in \mathcal{L}(n)$ and $P \in \operatorname{Sp}(n, \mathbb{R})$, then $P(V) \in \mathcal{L}(n)$, so $\operatorname{Sp}(n, \mathbb{R})$ acts on $\mathcal{L}(n)$.

In fact, it is easy to check that $\operatorname{span}\left[\begin{array}{c}I_{n} \\ K\end{array}\right] \in \mathcal{L}(n)$ if and only if $K^{\prime}=K$, provided that $K$ is an $n \times n$ matrix. From this fact, we can define $\phi: S(n) \rightarrow \mathcal{L}(n)$ by $\phi(K)=\operatorname{span}\left[\begin{array}{c}I_{n} \\ K\end{array}\right]$. Let $\mathcal{L}_{0}(n)$ consist of those subspaces in $\mathcal{L}(n)$ which are complementary to the $n$-dimensional subspace span $\left[\begin{array}{c}0 \\ I_{n}\end{array}\right]$. Then $\phi$ embeds the Euclidean space $S(n)$, the vector space of real symmetric $n \times n$ matrices, in $\mathcal{L}(n)$ as the open and dense subset $\mathcal{L}_{0}(n)$. Therefore, $\mathcal{L}(n)$ can be viewed as a compactification of $S(n)$.

As noted by many researchers (e.g. Hermann and Martin [14,15], Ammar and Martin [1], Martin [20], Shayman [23], etc.), the use of the Grassmann manifold in the theory of the Riccati equation is closely related to the use of the state-costate equations in the study of the Riccati equation.

We demonstrate this equivalence for the discrete-time Riccati equation (2.4), from which we simultaneously obtain the "discrete Riccati flow" and the extension of the discrete-time symplectic Riccati equation on $S(n)$ to $\mathcal{L}(n)$.

It is well-known (e.g. Badawi and Lindquist [2], Ammar and Martin [1]) that Riccati equation (2.4) can be written

$$
P_{t+1}=\left(M_{21}+M_{22} P_{t}\right)\left(M_{11}+M_{12} P_{t}\right)^{-1} \quad t \in \mathbb{Z}
$$

where $M_{i j}, i, j=1,2$ are submatrices of $M$

$$
M=\left[\begin{array}{cc}
F^{-T}+F^{-T} h g^{\prime} F^{-T} \rho^{-1} & -F^{-T} h h^{\prime} \rho^{-1} \\
g g^{\prime} F^{-T} \rho^{-1} & F-g h^{\prime} \rho^{-1}
\end{array}\right]
$$


where $\rho=1-h^{\prime} F^{-1} g$. We define a "discrete flow" on $\mathcal{L}(n)$ by $V\left(t, V_{0}\right)=M^{t}\left(V_{0}\right)$ for $t \in \mathbb{Z}$. Then, we have

$$
\phi\left(P\left(t, P_{0}\right)\right)=V\left(t, \phi\left(P_{0}\right)\right) \quad t \in \mathbb{Z}
$$

whenever $P\left(t, P_{0}\right)$ exists. In this manner $(2.4)$ is extended from $S(n)$ to the $\mathcal{L}(n)$. Let the partition $M^{t}$ be

$$
\left[\begin{array}{ll}
B_{11} & B_{12} \\
B_{21} & B_{22}
\end{array}\right]
$$

Then, it is not hard to verify that the solution of (2.4) with initial point $P_{0}$ is given by

$$
P\left(t, P_{0}\right)=\left(B_{21}+B_{22} P_{0}\right)\left(B_{11}+B_{12} P_{0}\right)^{-1} \quad t \in \mathbb{Z} .
$$

We want to prove that this formula is equivalent to (2.14), which relates the flow of the extended symplectic Riccati equation in discrete time on $\mathcal{L}(n)$ to the flow of $(2.4)$ on $S(n)$. The proof is a straightforward calculation.

$$
\begin{aligned}
V\left(t, \phi\left(P_{0}\right)\right) & =M^{t}\left(\operatorname{span}\left[\begin{array}{c}
I \\
P_{0}
\end{array}\right]\right) \\
& =\operatorname{span}\left(M^{t}\left[\begin{array}{c}
I \\
P_{0}
\end{array}\right]\right) \\
& =\operatorname{span}\left[\begin{array}{l}
B_{11}+B_{12} P_{0} \\
B_{21}+B_{22} P_{0}
\end{array}\right] \\
& =\operatorname{span}\left[\begin{array}{c}
I \\
\left(B_{21}+B_{22} P_{0}\right)\left(B_{11}+B_{12} P_{0}\right)^{-1}
\end{array}\right] \\
& =\phi\left(\left(B_{21}+B_{22} P_{0}\right)\left(B_{11}+B_{12} P_{0}\right)^{-1}\right) .
\end{aligned}
$$

Thus (2.14) is equivalent to the formula

$$
\phi\left(P\left(t, P_{0}\right)\right)=\phi\left(\left(B_{21}+B_{22} P_{0}\right)\left(B_{11}+B_{12} P_{0}\right)^{-1}\right) .
$$

Note that $\phi$ is injective, and hence, this is equivalent to (2.15).

The above discussion has shown that the discrete-time Riccati equation (2.4) can be viewed as a power iteration on the Lagrange-Grassmann manifold.

\section{HEURISTIC DISCUSSION}

Before further discussion, we like to give some examples in the case of second-order systems to illustrate how the dynamical system $(2.7)$ behaves when it starts with the initial values $(\alpha(0), \gamma(0))=:(\alpha, \gamma)$ corresponding to the situation where $D\left(z, z^{-1}\right)$ is sign indefinite on the unit circle. We call such pairs of $(\alpha, \gamma)$ sign indefinite initial data.

First, we look at a simple example which will demonstrate why we are interested in studying this nonclassical system-theoretic problem. To this end, we fix $\gamma_{0}$ and $\gamma_{1}$. Figure 1 shows the plane $\alpha \mapsto(\alpha, \gamma)$, where $\gamma_{0}=1 / 2$ and $\gamma_{1}=1 / 3$. Here the shaded regions correspond to the case when $D\left(z, z^{-1}\right)$ is sign definite on the unit circle, while the white regions consist of sign indefinite initial data. Each point in the bounded shaded region in Figure 1 corresponds to a positive real function $v(z)$, and hence to a bona fide stochastic system, and converges, by classical results, to a stable equilibrium $\left(\alpha_{\infty}, 0\right)$. However, as shown in [6] initial conditions in the four unbounded shaded regions also correspond to orbits which converge to stable or unstable equilibria $\left(\alpha_{\infty}, 0\right)$ except for a zero measure set which escape in finite time.

Figure 1 clearly illustrates an obvious fact: there is a large portion of parameters $\left(\alpha_{1}, \alpha_{2}\right)$ which do not converge to any equilibrium. A natural question is therefore: what happens with the dynamical system initialized 


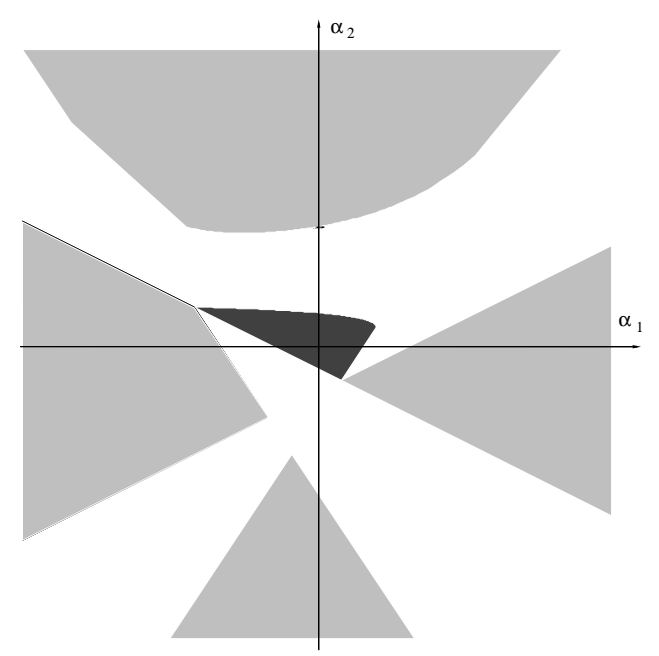

FIGURE 1. Initial data with $\left(\gamma_{0}, \gamma_{1}\right)=\left(\frac{1}{2}, \frac{1}{3}\right)$.

by such parameters? The objective of this paper is to understand the behavior of (2.7) corresponding to such initial data.

Intuitively, we have at least three kinds of behavior:

(i) $(\alpha, \gamma)$ is a periodic point;

(ii) the orbit of $(\alpha, \gamma)$ is dense on some manifold;

(iii) there is a finite-time escape.

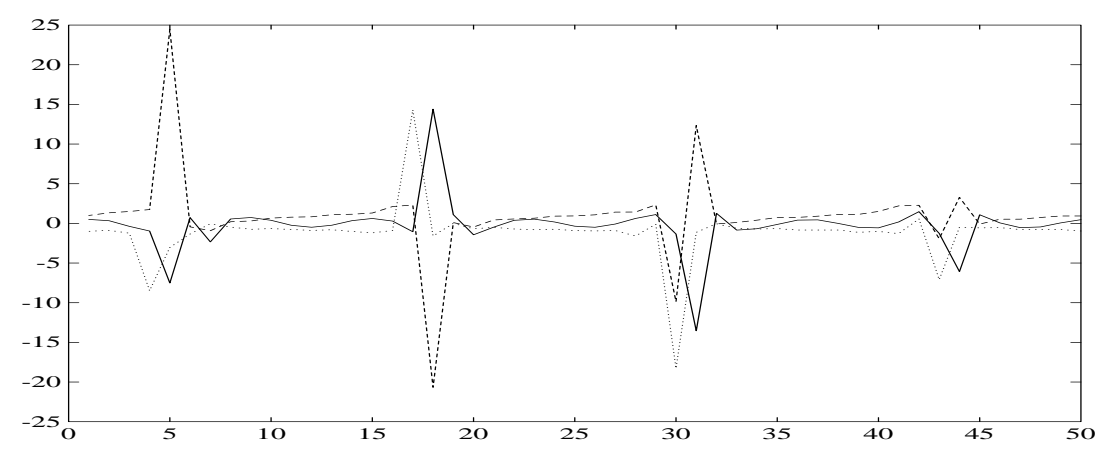

Figure 2. Trajectory of $\left(\alpha_{1}(t), \alpha_{2}(t), \gamma_{t}\right)$ initialized by $\left(-1,1, \frac{1}{2}, \frac{1}{3}\right)$.

For example, $\left(-\frac{9+3 \sqrt{2}+\sqrt{6 \sqrt{2}-5}}{6}, \frac{1+3 \sqrt{2}+\sqrt{6 \sqrt{2}-5}}{4}, \frac{1}{2}, \frac{1}{3}\right)$ is periodic of period 8 and $\left(-2,1, \frac{1}{2}, \frac{1}{3}\right)$ escapes in $t=4$ steps. A typical point of type (ii), say $\alpha=(-1,1)$, produces an orbit such as that in Figure 2, where we have plotted $\alpha_{1}(t), \alpha_{2}(t)$, and $\gamma_{t}=\gamma_{0}(t)$ for 50 iterations. Further, Figure 2 illustrates that the trajectories of $\left(\alpha_{1}(t), \alpha_{2}(t), \gamma_{t}\right)$ may have an "irregular" behavior when the initial points lie in the white region of Figure 1.

In [5], the complete analysis of the dynamics, for $n=1$, was carried out by studying the zero structure of the pseudo-polynomial $D\left(z, z^{-1}\right)$. With this in mind, the problem is reduced to investigating the zero structure of $D\left(z, z^{-1}\right)$ for an arbitrary $n$. To illustrate this point, we start with $n=2$. 


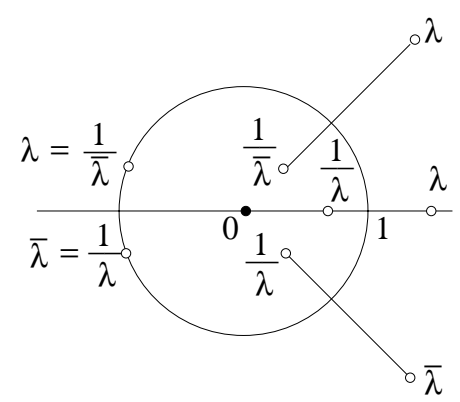

FiguRE 3. Zero structure of $D\left(z, z^{-1}\right)$.

Recall the fact that, because of the symplectic structure, the zeros of $D\left(z, z^{-1}\right)$ come in 4-tuples

$$
\lambda, \quad \bar{\lambda}, \frac{1}{\lambda}, \quad \frac{1}{\bar{\lambda}} \quad(|\lambda| \neq 1, \operatorname{Im} \lambda \neq 0)
$$

and pairs lying on the real axis

$$
\lambda=\bar{\lambda}, \frac{1}{\lambda}=\frac{1}{\bar{\lambda}},
$$

or on the unit circle

$$
\lambda=\frac{1}{\bar{\lambda}}, \quad \bar{\lambda}=\frac{1}{\lambda} .
$$

It is clear that in case $n=2$, we have, generically, the zero structures shown in Figure 4.

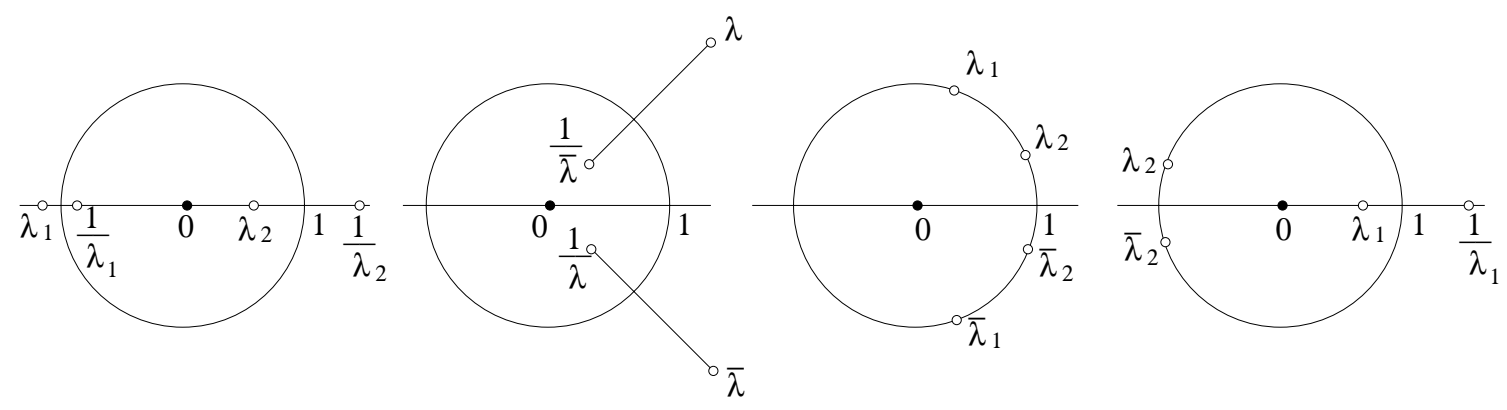

Figure 4. Zero location of $D\left(z, z^{-1}\right)$ for $n=2$.

By genericity, we mean that the "degree of freedom" for the zero structure is equal to the order of systems under consideration. Apparently, this is equivalent to saying that all zeros are simple. To see why these cases are called generic, we look at Figure 5. We now move the point $P$ along the line $l$ in same direction as shown in the figure. The root loci of $D\left(z, z^{-1}\right)$ corresponding to the parameters in the segments $P_{1} P_{2}$ and $P_{3} P_{4}$ are depicted in Figure 6.

We see that, in most cases, the zero structure of $D\left(z, z^{-1}\right)$ is like those shown in Figure 4. However, it also happens that the number of zeros decrease to 2 or 1 , with multiplicity 2 or 4 , which we call nongeneric. From this illustration, we might say that a zero $\lambda$ of $D\left(z, z^{-1}\right)$ can leave the unit circle only by colliding with another zero; at the same time, the complex-conjugate zeros will collide, and from the two pairs of zeros on the unit circle we obtain one 4-tuple (or pair of real $\lambda$ ), see Figure 6. 


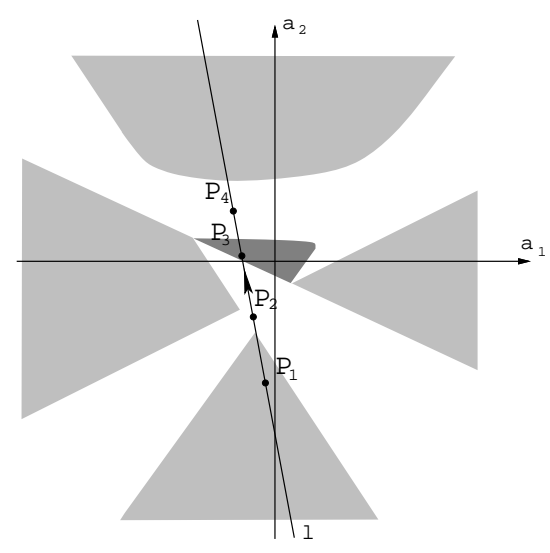

FiguRe 5
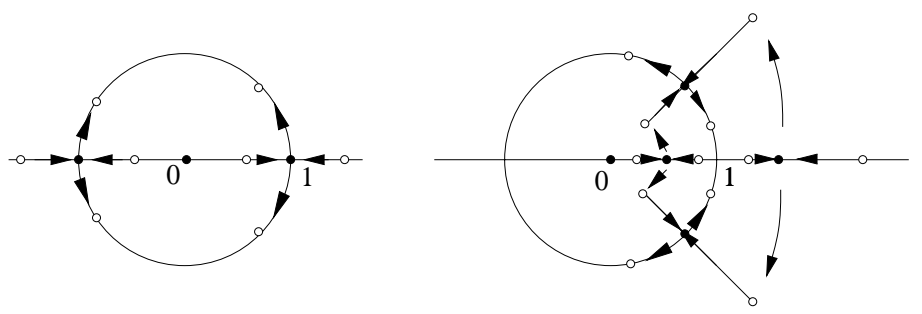

FiguRE 6. Zeros corresponding to $\alpha$ on $l$.

Dynamical behavior corresponding to the first two cases in Figure 4 has been analyzed completely [6]. To study the dynamical behavior of (2.7) initialized by the sign indefinite parameters remains unclear.

\section{Description of the phase portraits}

In [5], it was shown that in the case $n=1$ the invariant sets of the dynamical system can be expressed explicitly as a pair of hyperbola. This certainly provides a very elegant framework for analyzing the dynamical system under study. No doubt the analysis of orbits of higher order systems will be much harder. Even for a second-order system, the description of the invariant set is already rather involved. This is mainly due to the fact that the zero structure of $D\left(z, z^{-1}\right)$ is more complicated, as for the matrix Riccati equation, also to the incompletely understood geometric structure of the subvariety of $G^{n}\left(\mathbb{R}^{n+m}\right)$ consisting of all $n$-dimensional invariant subspaces under certain action.

In this section, we shall study the properties of the trajectory of the dynamical system (2.7) when the initial condition $(\alpha, \gamma)$ is such that $D$ is sign indefinite. By Lemma 2.1, there exists at least one nonzero coefficient among $d_{i}, i=0,1, \ldots, n$, say, $d_{i_{0}} \neq 0$. We may describe $X_{D}$ introduced in Section 2 in terms of the functions $\mathbb{R}^{2 n} \rightarrow \mathbb{R}^{n}$ as

$$
h_{i}(\alpha, \gamma)=\frac{d_{i}(\alpha, \gamma)}{d_{i_{0}}(\alpha, \gamma)}, \quad 0 \leq i \leq n, i \neq i_{0}
$$

where $d_{i}(\alpha, \gamma)$ 's are defined in Lemma 2.1 . 
Theorem 4.1. Suppose that $(\alpha, \gamma)$ does not escape in finite time. Then, at each point of the trajectory,

$$
h_{i}(\alpha(t), \gamma(t))=\kappa_{i}, \quad 0 \leq i \leq n, i \neq i_{0}
$$

where $\kappa_{i}$ are constants which can be determined from the initial condition $(\alpha, \gamma)$. In fact, if $\alpha_{n} \neq 0$, then we may choose $i_{0}=n$, and

$$
d(z)=\alpha_{n}\left[z^{n}+\kappa_{n-1} z^{n-1}+\cdots+\frac{\kappa_{0}}{2}\right]
$$

and if $\alpha_{n}=\cdots=\alpha_{k+1}$, but $\alpha_{k} \neq 0$, then, we may choose $i_{0}=k$, and $\kappa_{n}=\cdots=\kappa_{k+1}=0, \kappa_{k} \neq 0$, and

$$
d(z)=\alpha_{k} r_{n-k}\left[z^{k}+\kappa_{k-1} z^{k-1}+\cdots+\frac{\kappa_{0}}{2}\right] .
$$

Conversely, any point $(\alpha, \gamma)$ such that

$$
h_{i}(\alpha, \gamma)=\kappa_{i} \quad i=0,1, \ldots, n-1
$$

has an orbit satisfying (4.2) and the same pseudo-polynomial $D\left(z, z^{-1}\right)$ modulo multiplication by a nonzero constant.

Proof. Since there is no finite escape time, $r_{t} \neq 0$ for all $t \in \mathbb{Z}$. In view of $(2.12)$ and $d_{i_{0}}(\alpha, \gamma) \neq 0$, we see that $d_{i_{0}}(\alpha(t), \gamma(t)) \neq 0$. Hence, the rational function (4.1) are finite on the whole trajectory. Moreover, for all $t \in \mathbb{Z}$,

$$
h_{i}(\alpha(t), \gamma(t))=h_{i}(\alpha(0), \gamma(0)), \quad i \neq i_{0}
$$

Setting $\kappa_{i}=h_{i}(\alpha(0), \gamma(0))$, we obtain (4.2). Next, note that $d_{n}=\alpha_{n}$ (Lem. 2.1), then, if $\alpha_{n} \neq 0, h_{i}(\alpha, \gamma)$ $=\frac{d_{i}(\alpha, \gamma)}{d_{n}(\alpha, \gamma)}, i=0,1, \ldots, n-1$, are well-defined, $d_{i}=\kappa_{i} \alpha_{n}$. Consequently (4.3) follows. If $\alpha_{n}=\cdots=\alpha_{k+1}=0$, but $\alpha_{k} \neq 0$, then, by Lemma $2.1, d_{i}=0$, for $i=k+1, \cdots, n$, and $d_{k}=r_{n-k} \alpha_{k} \neq 0$. Hence, $\kappa_{n}=\cdots$ $=\kappa_{k+1}=0$, and $d_{i}=\kappa_{i} a_{k}$, and hence, (4.4) follows. Consequently, any $(\alpha, \gamma) \in \mathbb{R}^{2 n}$ satisfying $h_{i}(\alpha, \gamma)=\kappa_{i}$, $i=0,1, \ldots, n-1$ has a pseudo-polynomial that differs from $D\left(z, z^{-1}\right)$ by at most the nonzero constant, $\alpha_{n}$ or $\alpha_{k} r_{n-k}$, whichever case applies, and therefore the points on its orbit satisfying (4.2).

From Theorem 4.1, we may call the invariant set $X_{D}$ the level sets of the functions $h_{i}$ 's. For the exposition simplicity, we shall assume, in the sequel, that the initial condition $(\alpha, \gamma)$ has the property that $\alpha_{n} \neq 0$ (for otherwise there is a dimension reduction, as shown in Th. 4.1) and $\gamma_{k}^{2} \neq 1, k=0,1, \ldots, n-1$ and that it corresponds to the situation where $D\left(z, z^{-1}\right)$ is sign indefinite on the unit circle. Further, we shall use the $(a, b)$ - and $(\alpha, \gamma)$-coordinate systems interchangeably, for the mapping $\mathcal{F}$ (see [7], Prop. 4.1), between them is a birational isomorphism.

Theorem 4.2. The level set $X_{D}$ is invariant under the dynamical system (2.7) and is an $(n-\sigma)$-submanifold in $\mathbb{P R}^{2 n}$, where $\sigma$ is the number of common pairs of reciprocal zeros of the polynomials a $(z)$ and b( $\left.z\right)$ given by (2.6). Moreover (2.7) is invariant under the transformation

$$
(\alpha, \gamma) \rightarrow(\alpha,-\gamma)
$$

Proof. Inspecting (2.7), it is clear that the dynamical system under discussion is invariant under the transformation defined in (4.5). The first part follows from Theorem 4.1, and the proof of $X_{D}$ being an $(n-\sigma)$-submanifold in $\mathbb{R}^{2 n}$ is analogous to ([7], Th. 4.15).

Next, we shall discuss the motion on the submanifold $X_{D}$. To this end, we need some notations and definitions. We consider the time evolution

$$
x_{t+1}=f\left(x_{t}\right), \quad t \in \mathbb{Z}
$$


where $f: \mathbb{R}^{N} \rightarrow \mathbb{R}^{N}$ is a differentiable vector function. Let $U \subset \mathbb{R}^{N}$ be an open subset. For an initial point $x_{0} \in U$, the forward trajectory

$$
\mathcal{O}\left(x_{0}\right)=\left\{x_{t} ; t=0,1,2, \ldots\right\}
$$

is called the orbit of $x_{0}$. A periodic orbit of $f$ is a set of points $\left\{x_{0}, x_{1}, \ldots, x_{p-1}\right\}$ such that (4.6) holds for $i=0,1, \ldots, p-2$ and $f\left(x_{p-1}\right)=x_{0}$. We call $p$ the minimum period (period in short) of the periodic orbit.

Let us turn to the motion on the invariant level set $X_{D}$. For simplicity we make one more assumption, namely, that the polynomials $a(z)$ and $b(z)$ have no common pairs of reciprocal roots. Then $X_{D}$, defined by (4.1), is an $n$-submanifold generated by sign indefinite initial conditions $\alpha$ and $\gamma$. Next theorem describes the dynamical behavior of (2.7), in the simplest but most generic situation.

Theorem 4.3. Let $(\alpha, \gamma)$ be sign indefinite initial data. Assume that $D\left(z, z^{-1}\right)$ has $2 k$ simple zeros, $e^{ \pm i \theta_{1}}$, $e^{ \pm i \theta_{2}}, \ldots, e^{ \pm i \theta_{k}}, k \leq n$ on the unit circle, and the rest of zeros (if any) can be real or of even multiplicity on the unit circle. Then, under the dynamics $(2.7),(\alpha, \gamma)$ either escapes in a finite number of steps, or generates

(i) a periodic orbit on the $k$-submanifold $X_{D}$, if $\theta_{1}, \theta_{2}, \ldots, \theta_{k}$ are rational multiples of $\pi$, or

(ii) a dense orbit on the $k$-submanifold $X_{D}$, if $\theta_{1}, \theta_{2}, \ldots, \theta_{k}, \pi$ are linearly independent over the rationals, or

(iii) an orbit on the $k$-submanifold $X_{D}$ which is neither dense nor periodic, if $\theta_{1}, \theta_{2}, \ldots, \theta_{k}$, $\pi$ are linearly dependent over the rationals, but not all the $\theta$ 's are rational multiples of $\pi$.

Obviously, $k=n$ is an extreme case of sign indefiniteness of $D\left(z, z^{-1}\right)$, i.e. all zeros are simple and on the unit circle. This is the only case appearing in the first-order dynamical system. Following Theorem 4.3, we may conclude that we are able to completely describe the phase portrait of the second-order fast filtering algorithm. The discussion of the zero structure in Section 3 illustrates this fact best. In this case, $D\left(z, z^{-1}\right)$ has

(a) four simple unimodular zeros of $D\left(z, z^{-1}\right)$;

(b) $1(-1)$ of multiplicity 2 is a zero and a pair of complex conjugate zeros on the unit circle;

(c) two real zeros and a pair of complex conjugate zeros on the unit circle.

The invariant set $X_{D}$ is described by, if $\alpha_{2} \neq 0$,

$$
\left\{\begin{aligned}
\left(r_{1} \alpha_{1}+\alpha_{1} \alpha_{2}+\gamma_{0} \gamma_{1} \alpha_{2}\right) & =\kappa_{1} \alpha_{2}^{2} \\
\left(\alpha_{2}^{2}+r_{1} \alpha_{1}^{2}+r_{2}\right) & =\kappa_{0} \alpha_{2}
\end{aligned}\right.
$$

where $r_{1}=1-\gamma_{0}^{2}$ and $r_{2}=\left(1-\gamma_{0}^{2}\right)\left(1-\gamma_{1}^{2}\right)$. The simulations in Figure 7 and Figure 8 illustrate case (a). Figure 7 corresponds to the motion described in (ii) of Theorem 4.3 and Figure 8 to that in (iii). Here we used the fact that the Schur parameters are updated according to the recursion (2.7a). So we could plot the phase portraits in the three-dimensional space in a natural way.

The next two pictures illustrate that, in cases (b) and (c), $\left(\alpha_{t}, \gamma_{t}\right)$ converges to a 1-submanifold on $X_{D}$. In the present examples the trajectories are dense on 1-submanifolds.

If $\alpha_{2}=0$, but $\alpha_{1} \neq 0$, it is not hard to see that (2.7) contains two equations, and therefore $X_{D}$ is described by a pair of hyperbola. The phase portrait in this case was given in [5].

\section{Proof of Theorem 4.3}

The aim of this section is to prove Theorem 4.3 stated in the previous section. As before, our analysis is based on the dynamical behavior of the related matrix Riccati equation. As a consequence of our proofs, we shall see that the dynamical behavior of the Kalman gain sequence $\left\{k_{t}\right\}$ is topologically equivalent to the dynamical behavior of the fast filtering algorithm.

Let us recall some results from earlier work. In [7], it was shown that the fast filtering algorithm (2.7) tends to a limit $\left(\alpha_{\infty}, 0\right)$ if and only if the related Riccati equation (2.4) with initial condition $P_{0}=0$ converges to 


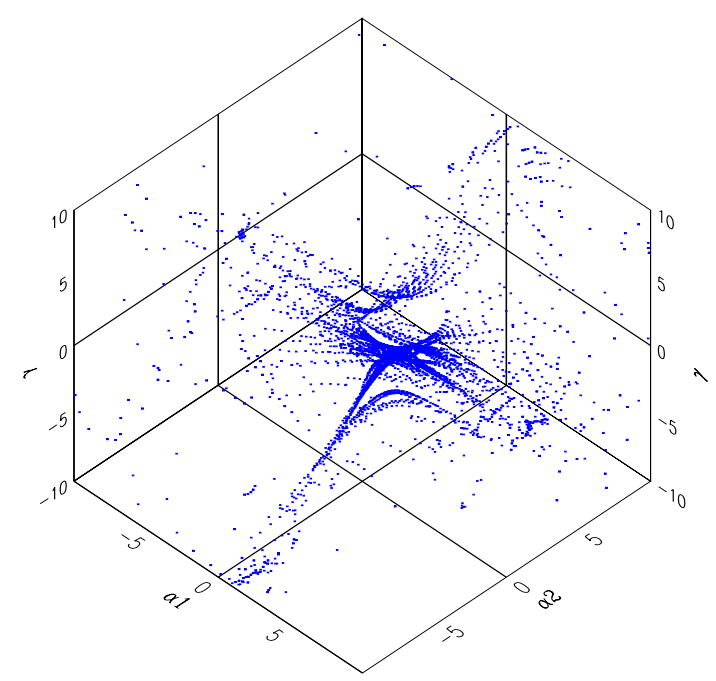

FIGURE 7

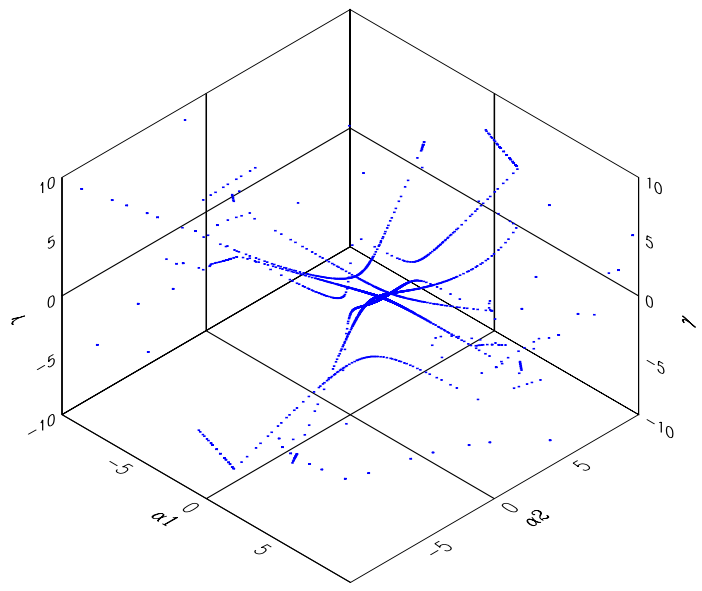

FIGURE 8

some equilibrium $P_{\infty}$. This shows that the nonconvergence of one implies the nonconvergence of the other and vice versa. Moreover (2.7) escapes in finite time if and only if (2.4) does. Hence, if $(\alpha, \gamma)$ does not generate an unbounded trajectory, then $X_{t}$ is nonsingular for all $t \in \mathbb{Z}$, where $X_{t}$ is computed through the symplectic 


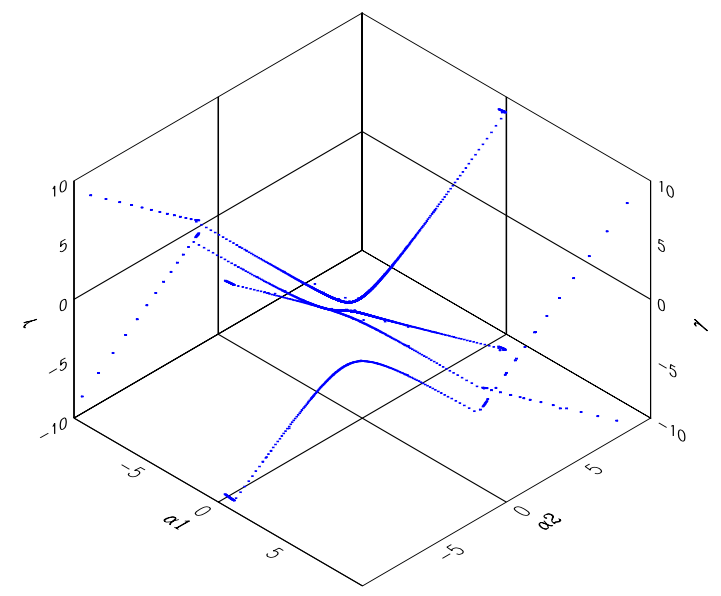

Figure 9. Orbit generated by $\left(\frac{1}{2}, \frac{1}{3},-\frac{1}{3},-\frac{5}{6}\right)$, case (b).

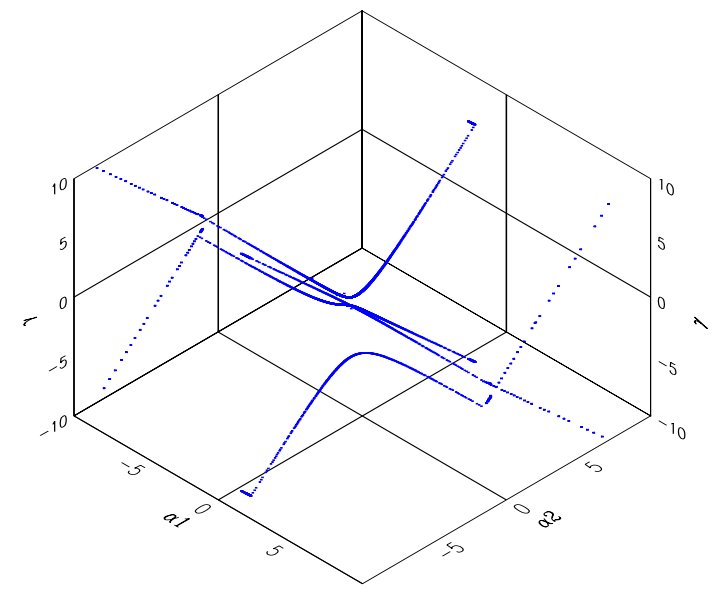

Figure 10. Orbit generated by $\left(\frac{1}{2}, \frac{1}{3},-\frac{1}{2},-\frac{3}{4}\right)$ case (c).

system

$$
\left[\begin{array}{c}
X_{t+1} \\
Y_{t+1}
\end{array}\right]=M\left[\begin{array}{c}
X_{t} \\
Y_{t}
\end{array}\right], \quad\left[\begin{array}{c}
X_{0} \\
Y_{0}
\end{array}\right]=\left[\begin{array}{l}
I \\
0
\end{array}\right]
$$

where the symplectic matrix $M$ is defined in (2.13). Furthermore, we know that the characteristic polynomial of $M$ and the pseudo-polynomial $D$ have the relation

$$
z^{n} D\left(z, z^{-1}\right)=\alpha_{n} \chi_{M}(z)
$$


Hence, the zeros of $D$ are eigenvalues of $M$ with same multiplicity.

\subsection{All simple unimodular zeros}

We first consider the symplectic system defined by (5.1), i.e. $Z_{t+1}=M Z_{t}$ with initial value $Z_{0}=\left[\begin{array}{c}I \\ P_{0}\end{array}\right]$. First, let $k=n$. Then, $D\left(z, z^{-1}\right)$ has $2 n$ distinct zeros on the unit circle, which implies that $M$ has all simple eigenvalues on the unit circle. So $e^{ \pm i \theta_{1}}, \ldots, e^{ \pm i \theta_{n}}$ are the $2 n$ eigenvalues of $M$ defined in (2.13). Let $V_{k}, k=1, \ldots, n$ denote primary components of $M$ corresponding to $e^{i \theta_{k}}, k=1, \ldots, n$, and let $G^{1}\left(V_{k}\right)$ be the Grassmann manifold of all 1-dimensional subspaces of $V_{k}$. Now we want to study the set $\mathcal{T}:=\left\{W_{1} \oplus W_{2} \oplus \cdots \oplus\right.$ $\left.W_{n}: W_{k} \in G^{1}\left(V_{k}\right)\right\}$.

Proposition 5.1. $\mathcal{T}$ is an $n$-torus, and it is invariant under the dynamical system (5.1).

Proof. First we have a simple fact that $\operatorname{dim} V_{k}=2, k=1, \ldots, n$. Then $\mathcal{T}$ is isomorphic to the product

$$
G^{1}\left(V_{1}\right) \times \cdots \times G^{1}\left(V_{n}\right) \simeq \underbrace{\mathbb{R \mathbb { P } ^ { 1 } \times \cdots \times \mathbb { R P } ^ { 1 }}}_{n \text { times }} \simeq \underbrace{S^{1} \times \cdots \times S^{1}}_{n \text { times }} \simeq \mathbb{T}^{n} .
$$

Thus, $\mathcal{T}$ is an $n$-torus.

Let $W \in \mathcal{T}$. Then, $W=W_{1} \oplus W_{2} \oplus \cdots \oplus W_{n}$ with $W_{k}$ a one-dimensional subspace of $V_{k} . \quad M^{t}(W)$ $=M^{t} W_{1} \oplus M^{t} W_{2} \oplus \cdots \oplus M^{t} W_{n}$ belongs to $\mathcal{T}$, since $W_{k} \subset V_{k}$ and $V_{k}$ is $M$-invariant. Thus, the torus $\mathcal{T}$ is invariant under the linear symplectic system (5.1).

Proposition 5.2. $\mathcal{T}$ is Lagrangian.

To prove Proposition 5.2, we need following result by Laub and Meyer [17].

Lemma 5.3. Let $M \in \operatorname{Sp}(n, \mathbb{R})$ have $2 n$ distinct eigenvalues on the unit circle. Then there exists a $T \in \operatorname{Sp}(n, \mathbb{R})$ such that $T M T^{-1}$ has the form

$$
\hat{M}=\left[\begin{array}{ll}
\hat{M}_{11} & \hat{M}_{12} \\
\hat{M}_{21} & \hat{M}_{22}
\end{array}\right]
$$

where

$$
\begin{aligned}
& \hat{M}_{11}=\hat{M}_{22}=\operatorname{diag}\left(\cos \theta_{1} t, \ldots, \cos \theta_{n} t\right) \\
& \hat{M}_{12}=-\hat{M}_{21}=\operatorname{diag}\left(\sin \theta_{1} t, \ldots, \sin \theta_{n} t\right)
\end{aligned}
$$

and the real submatrix $\left[\begin{array}{cc}\cos \theta_{k} & \sin \theta_{k} \\ -\sin \theta_{k} & \cos \theta_{k}\end{array}\right]$ is a canonical block analogous to the usual Jordan blocks. More precisely, $T$ has the form

$$
T=\left[q_{1}, q_{2}, \ldots, q_{n}, p_{1}, p_{2}, \ldots, p_{n}\right]
$$

where $q_{k}=\sqrt{2}\left(\operatorname{Re} v_{k}\right), p_{k}=-\sqrt{2}\left(\operatorname{Im} v_{k}\right), k=1, \ldots, n$, and $v_{k}$ is a basis for eigenspace corresponding to $e^{i \theta_{k}}$, with $v_{k} J \bar{v}_{k}= \pm i$.

From Lemma 5.3 we see that $\left\{q_{k}, p_{k}\right\}$ is a basis of $V_{k}$.

Proof of Proposition 5.2. Let $W$ be a subspace of $\mathcal{T}$ with base $\left[W_{1}, \ldots, W_{n}\right]:=U$. We shall prove that $U^{\prime} J U=0$. By the definition of $\mathcal{T}$, each $W_{k}$ can be represented by $q_{k}$ and $p_{k}$. Then, $U=\left[\xi_{1} q_{1}+\zeta_{1} p_{1}, \ldots, \xi_{n} q_{n}+\zeta_{n} p_{n}\right]$. By Lemma $5.3, q_{j}^{\prime} J p_{j}=1$, for $j=1, \ldots, n, q_{i}^{\prime} J q_{j}=p_{i}^{\prime} J p_{j}=0, i, j=1, \ldots, n$, and $q_{i}^{\prime} J p_{j}=0, i \neq j$. So the off-diagonal entries of $U$ are zeros, and the diagonal ones are $\xi_{i} \zeta_{i}\left(q_{i}^{\prime} J p_{i}+p_{i}^{\prime} J q_{i}\right) i=1, \ldots, n$. But $J$ is antisymmetric. Hence, $U^{\prime} J U=0$, proving the Lagrangian.

Now we turn to analyzing the trajectory of the Riccati equation (2.4) with initial value $P_{0}=0$. 
Theorem 5.4. Let the symplectic matrix $M$ defined in (2.13) have $n$ distinct complex conjugate pairs eigenvalues, $e^{ \pm i \theta_{k}}, k=1, \ldots, n$, and let $\phi$ be defined as in Section 2. Then, under the dynamics $(2.4)$, $P_{0}=0$ either escapes in finite time, or generates

(i) a periodic orbit on the $n$-torus $\phi^{-1}(\mathcal{T})$, if $\theta_{1}, \theta_{2}, \ldots, \theta_{n}$ are rational multiples of $\pi$, or

(ii) a dense orbit on the $n$-torus $\phi^{-1}(\mathcal{T})$ if $\theta_{1}, \theta_{2}, \ldots, \theta_{n}, \pi$ are linearly independent over the rationals, or

(iii) an orbit on the $n$-torus $\phi^{-1}(\mathcal{T})$ which is neither dense nor periodic, if $\theta_{1}, \theta_{2}, \ldots, \theta_{n}, \pi$ are linearly dependent over the rationals, but not all the $\theta_{k}$ 's are rational multiples of $\pi$.

Proof. Since $r_{t}=1-h^{\prime} P_{t} h$ is not sign preserved for $t \in \mathbb{Z}_{+}, r_{t}$ may be zero for some $t$, which implies that $X_{t}$ may be singular for some $t$. Hence, the trajectory of the Riccati equation under discussion may escape in finite time.

Let $(\alpha, \gamma)$ be sign indefinite parameter such that $P_{0}$ does not escape in forward time. Then, studying the trajectory of (2.4) is equivalent to studying the dynamical system (5.1), on the $n$-torus $\mathcal{T}$, for $\mathcal{T}$ is an $n$-dimensional $M$-invariant Lagrangian subspace (Prop. 5.1, Prop. 5.2), and hence the phase portrait description for (5.1) on $\mathcal{T}$ does describe the phase portrait of the original Riccati equation.

Since we are concerned with the trajectory generated by $Z_{0}$, we need to check that $\operatorname{span} Z_{0}=\operatorname{span}\left[\begin{array}{l}I \\ 0\end{array}\right] \in \mathcal{T}$. By Lemma 5.3, $q_{k}$ and $p_{k}$ are the normalized real part, respectively, the normalized imaginary part of the complex base, $v_{k}, \bar{v}_{k}$, in $V_{k}$. It is easily seen that $Z_{0}$ can be represented by $\left[v_{1}, \ldots, v_{n}\right] Q+\left[\bar{v}_{1}, \ldots, \bar{v}_{n}\right] \bar{Q}$, which implies that $Z_{0}$ can be represented by the real part of the $v_{k}$ 's. This yields $\operatorname{span} Z_{0} \subset \operatorname{span}\left[q_{1}, \ldots, q_{n}\right]$, i.e. span $Z_{0} \in \mathcal{T}$.

We turn to analyzing the trajectories of the dynamical systems $(5.1)$ on the $n$-torus $\mathcal{T}$. The isomorphism discussed above identifies $W \in V$ with $\left(W_{1}, \ldots, W_{n}\right) \in G^{1}\left(V_{1}\right) \times \cdots \times G^{1}\left(V_{n}\right)$ and the trajectories of the dynamical system (5.1), $M^{t}(W)$, with $\left(M^{t}\left(W_{1}\right), \ldots, M^{t}\left(W_{n}\right)\right)$. According to Kronecker [13], the motion $M^{t}\left(W_{k}\right)$ traverses the circle $S^{1}$ periodically if $\theta_{k}$ is rational multiple of $\pi$, or densely if $\theta_{1}, \ldots, \theta_{n}, \pi$ are linearly independent over the rationals. In particular, if all $\theta_{1}, \ldots, \theta_{n}$ are rational multiples of $\pi, \operatorname{sp}\left[\begin{array}{l}I \\ 0\end{array}\right]$ generates a periodic orbit on the $n$-torus $\mathcal{T}$ with period $T$, a natural number, which will be determined explicitly. To see this, let $\theta_{k}=\frac{\nu_{k}}{\delta_{k}} \pi$. Recalling that $e^{i \theta_{k}}$ and $e^{i\left(\theta_{k}+\pi\right)}$ are antipodal points on the same unit circle $S^{1}$, the projective line, we see that $M^{p_{k}} W_{k}=W_{k}$ for all $W_{k}$ in $V_{k}, k=1, \ldots, n$, and that $p_{k}$ is the smallest natural number with this property provided that $\nu_{k}, \delta_{k}$ are coprime, i.e. $\left(\nu_{k}, \delta_{k}\right)=1$. Hence (5.1) has an periodic orbit (on $\left.\mathcal{T}\right)$ with period $p$, where $p$ is the least common denominator of $\left\{\frac{\delta_{1}}{\nu_{1}}, \ldots, \frac{\delta_{n}}{\nu_{n}}\right\}$. By Kronecker again, sp $\left[\begin{array}{l}I \\ 0\end{array}\right]$ generates a dense orbit on the $n$-torus $\mathcal{T}$ if $\theta_{1}, \ldots, \theta_{n}, \pi$ are linearly independent over the rationals. Apparently, if $\theta_{k}, \pi, k=1, \ldots, n$, are linearly dependent over the rationals but not all $\theta_{k}$ 's are rational multiple of $\pi$, then the orbit of (5.1) is neither dense nor periodic on $\mathcal{T}$.

Proof of Theorem 4.3. Since $r_{t}$ is not sign preserved, an orbit starting at $(\alpha, \gamma)$ might escape in a finite number of steps, under the action of the dynamical system (2.7). Now assume that the $(\alpha, \gamma)$ does not escape in finite steps.

If $\mathcal{O}(0)=\left\{P_{t} ; t=0,1,2, \ldots\right\}$ is a period orbit of period $p$, then it is not hard to see that $r_{t+p}=r_{t}$, and $k_{t+p}=k_{t}$, because of equations $r_{t}=1-h^{\prime} P_{t} h$ and (2.3). Hence, $\gamma_{t+p}=\gamma_{t}$. Combining (2.5) with (2.6) yields

$$
\left[\begin{array}{c}
1 \\
k_{t}+a
\end{array}\right]=\left[\begin{array}{c}
1 \\
\frac{a(t)+b(t)}{2}
\end{array}\right]=\frac{1}{2}[\Psi(\gamma(t))+\Phi(\gamma(t))]\left[\begin{array}{c}
1 \\
\alpha(t)
\end{array}\right] .
$$

Again by the fact that $k_{t}$ and $\gamma_{t}$ are periodic of period $p$, we obtain that $\alpha(t+p)=\alpha(t)$. Thus,

$$
\mathcal{O}((\alpha, \gamma))=\{(\alpha(t), \gamma(t)) ; t=0,1,2, \ldots,\}
$$

is also periodic. Since $(\alpha(t), \gamma(t))$ is on the $n$-submanifold $X_{D}$, for all $t \in \mathbb{Z}_{+}, \mathcal{O}((\alpha, \gamma))$ is a periodic orbit on $n$-submanifold $X_{D}$.

Now we need to show that the orbit $\mathcal{O}((\alpha, \gamma))$ is dense on the $n$-submanifold $X_{D}$, if $\mathcal{O}(0)$ is dense on $\mathcal{T}$. By Theorem 5.4, it is not hard to see that $\left\{\operatorname{graph}\left(P_{t}\right)\right\}_{0}^{\infty}$ is dense, and consequently $\left\{P_{t} h ; t=0,1,2, \ldots\right\}$ is dense in $\mathbb{R}^{n}$. 
In view of (2.3), we see by $\alpha_{n} \neq 0$, that the correspondence between $k_{t}$ and $P_{t} h$ is one-to-one. Hence, $\left\{k_{t} ; t=0,1, \ldots\right\}$ is dense in $\mathbb{R}^{n}$. Moreover, since $D\left(z, z^{-1}\right)$ has only simple zeros, $a(z)$ and $b(z)$ have no common reciprocal zeros. So we know that $S(a)$ invertible (Th. 4.1), where

$$
S(a) b=a(z) b(1 / z)+a(1 / z) b(z) .
$$

Again, by (2.3), we have

$$
b_{t}=2 \tilde{k}_{t}-a_{t}
$$

where $\tilde{k}_{t}:=k_{t}+a$. We drop the index for simplicity. Using (5.2) gives

$$
S(a)(2 \tilde{k}-a)=2 D .
$$

Let us define $F(a, \tilde{k}):=S(a)(2 \tilde{k}-a)-2 D$. Then, $F(a, \tilde{k})=0$. A simple calculation shows that $\operatorname{deg} \operatorname{Jac} F=n$ at all points, because $\frac{\partial F_{i}}{\partial \tilde{k}_{j}}=S(a)$, which is invertible. Therefore, by implicit function theorem, there exists a unique continuous function $\mathcal{G}: \mathbb{R}^{n} \rightarrow \mathbb{R}^{n}$ such that $a=\mathcal{G}(\tilde{k})$. Obviously, $\mathcal{G}$ is densely onto. Since $a \in X_{D}, b$ is also uniquely determined by $b=2(S(\mathcal{G}(\tilde{k})))^{-1} D$, So the function $\mathbb{R}^{n} \rightarrow X_{D}$ is densely onto. Thus, we have proved that $\left\{\left(a_{t}, b_{t}\right) ; t=0,1, \ldots\right\}$ is dense on $X_{D}$.

Finally, combining both arguments for periodicity and denseness, we can conclude that the if $\mathcal{O}(0)$ is neither dense nor periodic orbit, $\mathcal{O}((\alpha, \gamma))$ is neither dense nor periodic on $X_{D}$.

\section{2. $k<n$ pairs of simple unimodular zeros}

In this case, we have following possibilities of zero structure: $2 k$ simple unimodular zeros, $e^{ \pm i \theta_{1}}, \ldots, e^{ \pm i \theta_{k}}$, and (i) real zeros which are not 1 or -1 (counted by multiplicity), or (ii) unimodular zeros of even multiplicity, where 1 and -1 are automatically of even multiplicity by the special property of $M$ considered here, or (iii) a combination of (i) and (ii).

We first discuss the dynamical behavior of the matrix Riccati equation. Let $v_{1} \ldots, v_{k}, \bar{v}_{1} \ldots, \bar{v}_{k}$, be the eigenvectors belonging to the $2 k$ simple unimodular eigenvalues, and $\tilde{v}_{2 k+1}, \ldots, \tilde{v}_{2 n}$, ordered as in ([7], Lem. 6.10), be the (generalized) eigenvectors belonging to the remaining eigenvalues. Then, $\operatorname{span}\left\{v_{1}, \ldots, v_{k}, \bar{v}_{1}, \ldots, \bar{v}_{k}\right\}$ is orthogonal to $\operatorname{span}\left\{v_{2 k+1}, \ldots, v_{2 n}\right\}$. By Lemma 5.3 , we see that

$$
\mathbb{R}^{2 n}=\operatorname{sp}\left\{q_{1}, \ldots, q_{k}, p_{1}, \ldots, p_{k}\right\} \oplus \operatorname{sp}\left\{v_{2 k+1}, \ldots, v_{2 n}\right\}=: \mathcal{U}_{1} \oplus \mathcal{U}_{2} .
$$

Therefore, $M$ can be decomposed as $M_{1} \oplus M_{2}$ where $M_{1}=\left.M\right|_{u_{1}}$ and $M_{2}=\left.M\right|_{u_{2}}$, where the eigenvalues of $M_{1}$ are $e^{ \pm i \theta_{1}}, \ldots, e^{ \pm i \theta_{k}}$. Hence, $M^{t} \operatorname{sp} Z_{0}$ is decomposed into two parts: $M_{1}^{t} \operatorname{sp} Z_{0}^{(1)}$ and $M_{2}^{t} \operatorname{sp} Z_{0}^{(2)}$, where $Z_{0}^{(1)}:=\left[\begin{array}{l}I_{k} \\ 0_{k}\end{array}\right]$ and $Z_{0}^{(2)}:=\left[\begin{array}{l}I_{n-k} \\ 0_{n-k}\end{array}\right]$. For the first part, we apply the analysis of Section 5.1 and, for the second, the discussion used in the proof of Theorem 7.1 in [7]. Thus, apart from the possible finite escape time, the second part tends to an equilibrium as $t \rightarrow \infty$, and the first has one of the three possible behaviors described in Theorem 5.4. So $M^{t} \operatorname{sp} Z_{0}$ tends to a $k$-torus, described in Theorem 5.4, as $t$ tends to $\infty$, and the trajectory is dense on the $k$-torus. By the same argument used in the end of the proof for all simple unimodular zeros, we show that Theorem 4.3 holds also for $k<n$ simple unimodular zeros. This completes the proof of Theorem 4.3.

We want to close this section by a remark which implies that, in principle, Theorem 4.3 is true, even if the polynomials $a(z)$ and $b(z)$ have common pairs of reciprocal zeros.

Remark 5.5. It is easy to see that, if the polynomials $a(z)$ and $b(z)$ have common pairs of reciprocal zeros, then there is a zero-pole cancellation in $v(z)+v\left(z^{-1}\right)$. Hence, first we can study a lower order system, and then proceed as in the proof of ([7], Th. 7.1). However, the level set $X_{D}$ is not smooth. The dimension of $X_{D}$ 
is $n-\sigma$, where $\sigma$ is the number of common pairs of reciprocal zeros of $a(z)$ and $b(z)$. Theorem 4.3 remains the same if we replace $n$ by $n-\sigma$.

Finally, Remark 5.5 partially describes the dynamical behavior of the fast filtering algorithm in the special situation of unimodular zeros of multiplicity $2 k+1, k \geq 1$. That is if those odd multiple zeros are the common zeros of $a(z)$ and $b(z)$, due to dimensional reduction, there is no difficulty in our analysis. However, a complete description of the fast filtering algorithm for unimodular zeros of any odd multiplicity $\geq 3$ needs to be further investigated.

\section{Phase Portraits: Revisited}

We described, in Section 3, the phase portraits of the fast filtering algorithm in the case where $D\left(z, z^{-1}\right)$ is sign indefinite on the unit circle (Th. 4.3). It is natural to inquire what happens in the exceptional cases when the $\theta$ 's are linearly dependent over the rationals, namely, we want to further describe the phase portrait corresponding to the last situation in Theorem 4.3. Since our description of the orbit is based on the linear independence over the rationals, some results from number theory are needed.

Let $[x]$ denote the greatest integer $\leq x$, and $(x)$ the fractional part of $x$. These are standard notations in number theory (e.g. $[12,13,16,21])$. So $(x)=x-[x]$. The following examples illustrate our point of view.

Example 6.1. A discrete orbit on the torus $\mathbb{T}^{2}=S^{1} \times S^{1}$. Let $\Theta_{1}=\frac{2}{3}$, and $\Theta_{2}=e$. Hence, $\frac{2}{3}$, $e$, and 1 are linearly dependent over the rationals. The orbit $\left\{\left(t \Theta_{1}\right),\left(t \Theta_{2}\right)\right\}$ is illustrated by the two diagrams in Figure 11. The first shows the torus cut open. Gluing it together by usual identification, we get the second picture.
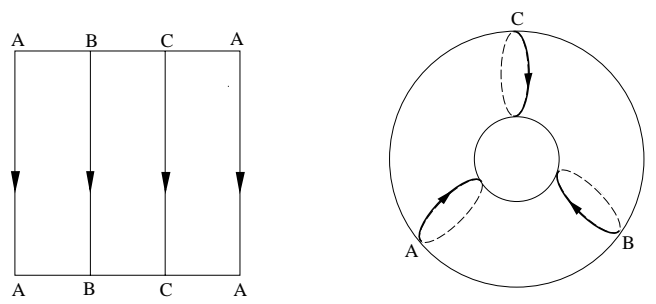

\section{FiguRE 11}

Figure 11 shows that $\left(\frac{2}{3}, e\right)$ does not generate a dense nor periodic orbit on $\mathbb{T}^{2}$. However, there are three subsequences which are dense on the three circles. The number 3 is determined by the rational $\Theta_{1}$.

Clearly, one can also have that both $\Theta$ 's are irrational but are linearly dependent on 1 over the rationals, for instance, $\Theta_{1}=3 \sqrt{2}-1$, and $\Theta_{2}=2-\sqrt{2}$. The orbit $\left\{\left(t \Theta_{1}\right),\left(t \Theta_{2}\right)\right\}$ is illustrated by the two diagrams in Figure 12. The first shows the torus cut open. Gluing it together by usual identification, we get the second picture.
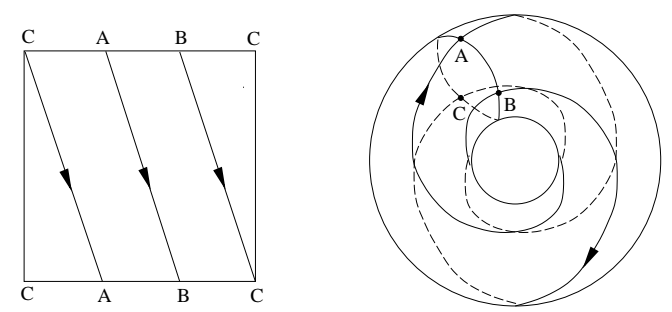

Figure 12 
From Figure 11 and Figure 12, we see that the orbits depicted in the unit squares look similar in the two cases, while after gluing the squares to the tori, the appearance of the orbits is quite different: the former lies on three disconnected circles, and the latter, on a closed curve on the torus. When we apply theses results to our dynamical system, we can also see a bit of the difference between the orbits in these two situations. See Figure 8 and Figure 13.

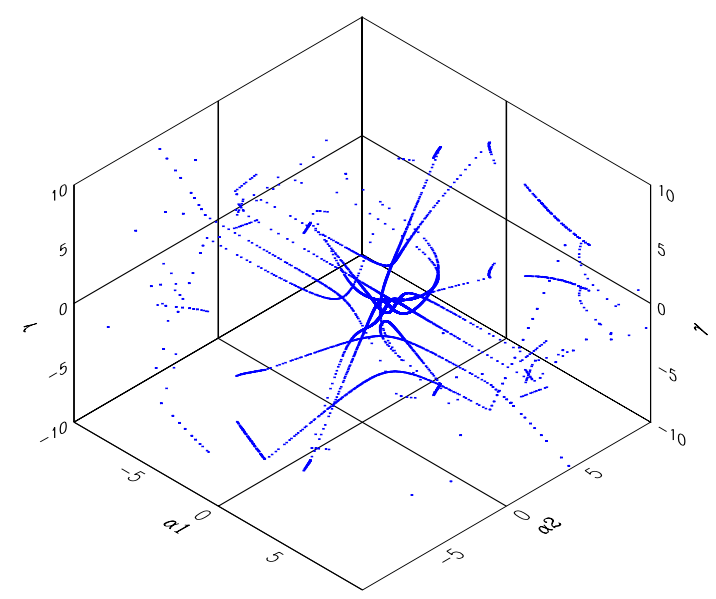

Figure 13

The mathematical statement of these phenomena and a geometric proof are given e.g. in Niven's book ([21], Chap. 3) We claim that this is also true for a more general setting. However, we first introduce the following lemma whose proof can be found e.g. ([8], p. 52).

Lemma 6.2. Let $\Theta_{j} \in \mathbb{R}$, for $j=1, \ldots, k$ not all be zero. There is a linearly independent (over the rationals) set of numbers $\Theta_{1}^{\prime}, \ldots, \Theta_{l}^{\prime}(l \leq k)$ such that each $\Theta_{j}$ is linearly dependent on $\Theta_{1}^{\prime}, \ldots, \Theta_{l}^{\prime}$.

Let $\mathbb{Q}$ be the set of all rationals. We shall consider the situation where the numbers $\Theta_{1}, \ldots, \Theta_{n}, 1$ are linearly dependent over $\mathbb{Q}$. The $\Theta^{\prime}$ 's are said to be connected by $n-l$ linear relations if there is a linearly independent $\left(\right.$ over $\mathbb{Q}$ ) set of numbers $\Theta_{1}^{\prime}, \ldots, \Theta_{l}^{\prime}(l \leq n)$ such that each $\Theta_{j}$ is linearly dependent (over $\mathbb{Q}$ ) on $\Theta_{1}^{\prime}, \ldots, \Theta_{l}^{\prime}, 1$.

The next theorem is, in fact, a companion result to Kronecker's theorem ([13], Chap. XXIII).

Theorem 6.3. Assume that $\Theta_{1}, \ldots, \Theta_{n}$ are connected by $n-l$ linear relations. Then, the points

$$
\left(\left(t \Theta_{1}\right),\left(t \Theta_{2}\right), \ldots,\left(t \Theta_{n}\right)\right), \quad t \in \mathbb{Z}_{+}
$$

lie on, and only on, those portions of the l-dimensional parallelograms lying within the $n$-unit "cube" $0 \leq \Theta_{j}<1$. Further, the points (6.1) are dense on these l-dimensional parallelograms.

As noted before, we distinguish two cases of the linear dependence: (a) some of the $\Theta$ 's are rational, (b) none of the $\Theta$ 's is rational. To situation (a), we give a definition for the sake of simplicity of exposition.

Definition 6.4. An orbit $\mathcal{O}\left(x_{0}\right)$ is said to be partially dense if there are $p$ subsequences $\left\{x_{p_{j}^{(k)}}\right\}_{j=1}^{\infty}=: \mathcal{O}\left(x_{p_{j}}^{(k)}\right)$, $k=1, \ldots, p, p_{j}^{(k-1)}<p_{j}^{(k)}$ such that every $\mathcal{O}\left(x_{p_{j}}^{(k)}\right)$ is dense on a submanifold of $U$.

We are now in a position to give the phase portraits of the dynamical system described by (2.7). To avoid excessively burdening our presentation with detail, we only discuss the case where all zeros of $D\left(z, z^{-1}\right)$ or 
eigenvalues of the symplectic matrix $M$ are distinct. Applying Theorem 6.3 to the Riccati equation (2.4) and a simple modification of the argument used in Section 5 , we can conclude

Theorem 6.5. Assume the hypotheses of Theorem 5.4 hold, and $P_{0}=0$ does not escape in finite time under (2.4). Then,

(a) $P_{0}$ generates a partially dense orbit on the $n$-torus $\phi^{-1}(\mathcal{T})$, if some of the $\theta$ 's are rational multiples of $\pi$, say, $\theta_{j}=\frac{\nu_{j}}{\delta_{j}} \pi, j=1, \ldots, k<n$, but $\theta_{j},(j=k+1, \ldots, n)$ and $\pi$ are linearly independent over $\mathbb{Q}$. Further, the trajectory $\left\{P_{t}\right\}$ is dense on the $p$ "slices" $(n-k)$-dimensional submanifold of the $n$-torus $\phi^{-1}(\mathcal{T})$, where $p$ is the least common denominator of $\left\{\frac{\delta_{1}}{\nu_{1}}, \ldots, \frac{\delta_{l}}{\nu_{l}}\right\}$,

or

(b) $P_{0}$ generates a dense orbit on an l-dimensional submanifold of the $n$-torus $\phi^{-1}(\mathcal{T})$, if $\theta$ 's are connected by $n-l$ linear relations and none of the $\theta$ 's is rational multiple of $\pi$.

Also we can prove a similar theorem for the fast filtering algorithm (2.7), by the argument in Section 5 .

Theorem 6.6. Assume the hypotheses of Theorem 4.3 hold, and $(\alpha, \gamma)$ does not escape in finite time under (2.7). Then, under the dynamics defined by (2.7),

(a) $(\alpha, \gamma)$ generates a partially dense orbit on the $k$-submanifold $X_{D}$, if some of the $\theta$ 's are rational multiples of $\pi$, say, $\theta_{j}=\frac{\nu_{j}}{\delta_{j}} \pi, j=1, \ldots, l<k$, but $\theta_{j},(j=l+1, \ldots, k)$ and $\pi$ are linearly independent over $\mathbb{Q}$. Further, $\left\{\left(\alpha_{t}, \gamma_{t}\right)\right\}$ is dense on the $p$ "slices" $(k-l)$ dimensional submanifold of the $k$-submanifold $X_{D}$, where $p$ is the least common denominator of $\left\{\frac{\delta_{1}}{\nu_{1}}, \ldots, \frac{\delta_{l}}{\nu_{l}}\right\}$,

or

(b) $(\alpha, \gamma)$ generates a dense orbit on an l-dimensional submanifold of the $k$-submanifold $X_{D}$, if $\theta$ 's are connected by $k-l$ linear relations and none of the $\theta$ 's is rational multiple of $\pi$.

\section{Phase portrait of the Discrete-time matrix Riccati equation}

The purpose of this section is to describe the complete phase portrait of

$$
P_{t+1}=F P_{t} F^{\prime}+\left(g-F P_{t} h\right)\left(1-h^{\prime} P_{t} h\right)^{-1}\left(g-F P_{t} h\right)^{\prime}
$$

with arbitrary initial value $P_{0}$. We shall see that the results presented here are parallel to those in [7] and previous sections.

Before further processing, we point out that a rather general discussion of the finite time escape for a more general Riccati equation can be found in Zhou [24].

Theorem 7.1. Consider the Riccati equation (7.1) where $\left(h^{\prime}, F\right)$ is observable, with initial condition $P_{0} \neq 0$, and let $M$ be defined by (2.13). Then, there is a finite escape time only if $P_{0} \notin \mathcal{P}_{-}$. For an initial condition $P_{0}$ which does not escape in finitely many steps,

(I) under the dynamics (7.1), $\left\{P_{t}\right\}$ converges to an equilibrium if and only if $M$ has no unimodular eigenvalues of odd multiplicity.

(II) If $M$ has $2 k$ simple unimodular eigenvalues, $e^{ \pm i \theta_{j}}, j=1, \ldots, k<n$, and no other unimodular eigenvalues of $M$ are of odd multiplicity, then, $P_{0}$, under the action of (7.1), generates either

(a) a period orbit on the $k$-torus $\phi^{-1}(\mathcal{T})$, if all $\theta$ 's are rational multiples of $\pi$, or

(b) a dense orbit on the $k$-torus $\phi^{-1}(\mathcal{T})$, if $\theta_{1}, \theta_{2}, \ldots, \theta_{k}$ and $\pi$ are linearly independent over $\mathbb{Q}$, or

(c) an orbit on the $k$-torus $\phi^{-1}(\mathcal{T})$ which is neither periodic nor dense, if $\theta_{1}, \theta_{2}, \ldots, \theta_{k}, \pi$ are linearly dependent over $\mathbb{Q}$, but not all the $\theta$ 's are rational multiples of $\pi$. More precisely, $P_{0}$ generates

(i) a partially dense orbit on the $k$-torus $\phi^{-1}(\mathcal{T})$, if some of $\theta$ 's are rational multiples of $\pi$, say, $\theta_{j}=\frac{\nu_{j}}{\delta_{j}} \pi, j=1, \ldots, l<k$, but $\theta_{j}, j=l+1, \ldots, k$, and $\pi$ are linearly independent. Further, $\left\{P_{t}\right\}$ 
is dense on the $p$ "slices" $(k-l)$-dimensional submanifold of the $k$-torus $\phi^{-1}(\mathcal{T})$, where $p$ is the least common denominator of $\left\{\frac{\delta_{1}}{\nu_{1}}, \ldots, \frac{\delta_{l}}{\nu_{l}}\right\}$, or

(ii) a dense orbit on an l-dimensional submanifold of the $k$-torus $\phi^{-1}(\mathcal{T})$, if the $\theta$ 's are connected by $k-l$ linear relations and none of the $\theta$ 's is rational multiple of $\pi$.

Proof. The assertion on finite escape follows from [24]. Let us assume that $P_{0}$ does not generate any unbounded trajectory. Without loss of generality, we study the canonical triple $(F, g, h)$ as defined in Section 2 . Now, we change variable $P$ to $\Sigma$ as follows: $P_{t}:=P_{0}-\Sigma_{t}$. Then the Riccati equation (7.1) can be written as

$$
\Sigma_{t+1}=F \Sigma_{t} F^{\prime}-\left(G+F \Sigma_{t} h\right)\left(r_{0}+h^{\prime} \Sigma_{t} h\right)^{-1}\left(G+F \Sigma_{t} h\right)^{\prime}+Q
$$

with $\Sigma_{0}=0$, where

$$
\begin{aligned}
Q & :=-\left(F P_{0} F^{\prime}-P_{0}\right), \\
G & :=-\left(F P_{0} h-g\right), \\
r_{0} & :=1-h^{\prime} P_{0} h .
\end{aligned}
$$

Note that (7.2) can be written

$$
\begin{aligned}
\Sigma_{t+1} & =\tilde{A} \Sigma_{t} \tilde{A}^{\prime}-\tilde{A} \Sigma_{t} h\left(r_{0}+h^{\prime} \Sigma_{t} h\right)^{-1} h^{\prime} \Sigma_{t} \tilde{A}^{\prime}+Q-G r_{0}^{-1} G^{\prime} \\
& =\tilde{A} \Sigma_{t} \tilde{A}^{\prime}-\tilde{A} \Sigma_{t} h\left(r_{0}+h^{\prime} \Sigma_{t} h\right)^{-1} h^{\prime} \Sigma_{t} \tilde{A}^{\prime}-\Lambda\left(P_{0}\right)
\end{aligned}
$$

where $\tilde{A}:=F-G r_{0}^{-1} h^{\prime}$.

By similar argument as before, we only need to study the dynamical behavior of the following system

$$
\left[\begin{array}{c}
X_{t+1} \\
Y_{t+1}
\end{array}\right]=\left[\begin{array}{cc}
\left(\tilde{A}^{\prime}\right)^{-1} & \left(\tilde{A}^{\prime}\right)^{-1} h r_{0}^{-1} h^{\prime} \\
-\Lambda\left(P_{0}\right)\left(\tilde{A}^{\prime}\right)^{-1} & \tilde{A}-\Lambda\left(P_{0}\right)\left(\tilde{A}^{\prime}\right)^{-1} h r_{0}^{-1} h^{\prime}
\end{array}\right]\left[\begin{array}{c}
X_{t} \\
Y_{t}
\end{array}\right]
$$

with $\left[X_{0}^{\prime}, Y_{0}^{\prime}\right]=[I, 0]$, where $\Lambda\left(P_{0}\right)$ defined as before.

We claim that (7.4) is topologically conjugate to the system

$$
\left[\begin{array}{c}
X_{t+1} \\
Y_{t+1}
\end{array}\right]=\left[\begin{array}{cc}
\left(A^{\prime}\right)^{-1} & -\left(A^{\prime}\right)^{-1} h h^{\prime} \\
g g^{\prime}\left(A^{\prime}\right)^{-1} & A+\left(1-\rho^{-1}\right) g h^{\prime}
\end{array}\right]\left[\begin{array}{c}
X_{t} \\
Y_{t}
\end{array}\right], \quad\left[\begin{array}{c}
X_{0} \\
Y_{0}
\end{array}\right]=\left[\begin{array}{l}
I \\
0
\end{array}\right]
$$

This can be shown by the identity

$$
\left[\begin{array}{cc}
I & 0 \\
P_{0} & -I
\end{array}\right]\left[\begin{array}{cc}
\left(A^{\prime}\right)^{-1} & -\left(A^{\prime}\right)^{-1} h h^{\prime} \\
g g^{\prime}\left(A^{\prime}\right)^{-1} & A+\left(1-\rho^{-1}\right) g h^{\prime}
\end{array}\right]\left[\begin{array}{cc}
I & 0 \\
P_{0} & -I
\end{array}\right]=\left[\begin{array}{cc}
\left(\tilde{A}^{\prime}\right)^{-1} & \left(\tilde{A}^{\prime}\right)^{-1} h r_{0}^{-1} h^{\prime} \\
-\Lambda\left(P_{0}\right)\left(\tilde{A}^{\prime}\right)^{-1} & \tilde{A}-\Lambda\left(P_{0}\right)\left(\tilde{A}^{\prime}\right)^{-1} h r_{0}^{-1} h^{\prime}
\end{array}\right]=: \tilde{M} .
$$

(This is a tedious but straightforward computation.) Hence, the dynamics of (7.4) is identical to that of (7.5). The latter has already been studied in [7] and in the preceding sections. From this identity, the symplectic matrices $\tilde{M}$ and $M$ have same eigenvalues of same multiplicity. Therefore, the theorem follows immediately from ([7], Th. 7.1), Theorem 5.4 and Theorem 6.5.

We close this section by a short comment. It is known [2] that there is a rank restriction on the operator $\Lambda\left(P_{0}\right)$ in order to apply fast filtering algorithm. However, Theorem 7.1 shows that the phase portraits of the related Riccati equation without this rank restriction can be described through that of the special initial condition. In this sense, Theorem 7.1 gives a more general result. 


\section{Conclusions}

In this paper, we have given a complete description for the phase portraits of the fast filtering algorithm and its related Riccati equation for an arbitrary initial value. Specially, we study the case where the pseudopolynomial (or the symplectic matrix related to Riccati equation) has zeros (or eigenvalues) on the unit circle. The key to describe the phase portrait is to utilize special structures the fast filtering algorithm offers, and thereafter, to associate with extended Riccati equation. This way, we were able to describe phase portraits for the Riccati equation related to fast filtering algorithms starting with an arbitrary initial value $P_{0}$. Here no rank restriction on the operator $\Lambda\left(P_{0}\right)$ is posed.

Let us briefly recapitulate the motion on an invariant manifold. If there is no zeros (eigenvalues, for the Riccati equation) of certain pseudo-polynomial (the symplectic matrix) on the unit circle, then the trajectory generated by the fast filtering algorithm (Riccati equation) converges to an equilibrium. Otherwise, the motion on the invariant manifold is neither periodic nor dense, in general, depending on whether or not the arguments, $\theta_{i}$, of associated zeros (eigenvalues) are rationally dependent. Notice that to formalize the filtering algorithm, it is assumed that $\operatorname{rank} \Lambda\left(P_{0}\right)=1$. However, using the fact that the extended special Riccati equation with arbitrary initial value is topological conjugate to that with 0 initial value, we can eventually drop the rank condition and derive the similar results for a more general type of equations. Although the motion is generally not dense on the whole invariant manifold, we can classify the situations in more detail by further discussion of the $\theta_{i}$ of associate zeros (eigenvalues). Roughly speaking, when $\theta_{i}$ are rationally dependent, but not all $\theta_{i}$ are rational multiple of $\pi$, we divide rational dependency into two parts: those with $\theta_{i}$ being rational multiple of $\pi$ and those linearly depended but not rational multiple of $\pi$.

In this paper, we have not dealt with the case where the unimodular zeros are odd multiple. We leave this as future research topic. Furthermore, we wish to extend the results in [7] and this paper to multi-output case.

Most of this work was complete when the author was a graduate student at Division of Optimization and Systems Theory, Royal Institute of Technology. The author would like to thank Professor Anders Lindquist for his professional advice, Professors Christopher I. Byrnes and Clyde Martin for their inspiring discussions and various constructive suggestions.

\section{REFERENCES}

[1] G. Ammar and C. Martin, The geometry of matrix eigenvalue methods. Acta Appl. Math. 5 (1986) 239-279.

[2] F.A. Badawi and A. Lindquist, A Hamiltonian approach to the factorization of the matrix Riccati equation. Math. Programming Stud. 18 (1982) 27-38.

[3] C.I. Byrnes, A. Lindquist, S.V. Gusev and S. Matee, A complete parametrization of all positive rational extensions of a covariance sequence. IEEE Trans. Automat. Control AC-40 (1995) 1841-1857.

[4] C.I. Byrnes and A. Lindquist, On the partial stochastic realization problem. IEEE Trans. Automat. Control AC-42 (1997) 1049-1070.

[5] C.I. Byrnes, A. Lindquist and T. McGregor, Predictability and unpredictability in Kalman filtering. IEEE Trans. Automat. Control 36 (1991) 563-579.

[6] C.I. Byrnes, A. Lindquist and Y. Zhou, Stable, unstable and center manifolds for fast filtering algorithms. Modeling, Estimation and Control of Systems with Uncertainty, G.B. Di Masi, A. Gombani and A. Kurzhanski, Eds., Birkhäuser Boston Inc. (1991).

[7] _ On the nonlinear dynamics of fast filtering algorithms. SIAM J. Control Optim. 32 (1994) 744-789.

[8] J.W.S. Cassels, An Introduction to Diophantine Approximation, Cambridge University Press, Cambridge (1956).

[9] H.J. Landau, C.I. Byrnes and A. Lindquist, On the well-posedness of the rational covariance extension problem, Tech. Report TRITA/MAT-96-OS5, Department of Mathematics, KTH, Royal Institute of Technology, Stockholm, Sweden (1996).

[10] S.V. Gusev, C.I. Byrnes and A. Lindquist, A convex optimization approach to the rational covariance extension problem, Tech. Report TRITA/MAT-97-OS9, Department of Mathematics, KTH, Royal Institute of Technology, Stockholm, Sweden (1997).

[11] P. Faurre, M. Clerget and F. Germain, Opérateurs Rationnels Positifs, Dunod (1979).

[12] G.H. Hardy and J.E. Littlewood, Some problems of Diophantine approximation. Acta Math. 37 (1914) $155-239$.

[13] G.H. Hardy and E.M. Wright, An Introduction to the Theory of Numbers, Oxford at the Clarendon Press (1954).

[14] R. Hermann and C. Martin, Lie and Morse theory for periodic orbits of vector fields and matrix Riccati equations, I: General Lie-theoretic methods. Math. Systems Theory 15 (1982) 277-284.

[15] $\ldots$ Lie and Morse theory for periodic orbits of vector fields and matrix Riccati equations. II. Math. Systems Theory 16 (1983) 297-306. 
[16] J.F. Koksma, Diophantische Approximationen, Chelsea Publishing Company, New York (1936).

[17] A.J. Laub and K. Meyer, Canonical forms for symplectic and Hamiltonian matrices. Celestial Mech. 9 (1974) $213-238$.

[18] A. Lindquist, A new algorithm for optimal filtering of discrete-time stationary processes. SIAM J. Control 12 (1974) $736-746$.

[19] Some reduced-order non-Riccati equations for linear least-squares estimation: the stationary, single-output case. Int. J. Control 24 (1976) 821-842.

[20] C. Martin, Grassmannian manifolds, Riccati equations, and feedback invariants of linear systems, Geometrical Methods for the Theory of Linear Systems, C.I. Byrnes and C. Martin, Eds., Reidel Publishing Company (1980) $195-211$.

[21] I. Niven, Diophantine Approximations, Interscience Publishers, New York, London (1956).

[22] I.R. Shafarevitch, Basic Algebraic Geometry, Springer-Verlag, Heidelberg (1974).

[23] M. Shayman, Phase portrait of the matrix Riccati equations. SIAM J. Control Optim. 24 (1986) 1-65.

[24] Y. Zhou, Monotonicity and finite escape time of solutions of the discrete-time riccati equation, to appear in proceedings of European Control Conference, Karlsruhe (1999). 